\title{
Semen analysis standardization: is there any problem in Polish laboratories?
}

\author{
Renata Walczak-Jedrzejowska ${ }^{1, *}$, Katarzyna Marchlewska ${ }^{2, *}$, Elzbieta Oszukowska ${ }^{1}$, Eliza Filipiak ${ }^{2}$, \\ Leszek Bergier ${ }^{3}$ and Jolanta Slowikowska-Hilczer ${ }^{2}$
}

\begin{abstract}
The aim of the study was to determine the degree of compliance of Polish laboratories with World Health Organization (WHO) recommendations, with regard to semen analysis methodology. A survey requesting information about methods of semen analysis was distributed to employees of 55 laboratories. Respondents who had participated in external seminological workshops (31\%) were termed certified respondents (CR), the remaining (69\%)—non-certified respondents (NCR). Only one laboratory (6\%) in the CR group and none in the NCR were compliant with WHO guidelines for methods and equipment used to evaluate seminal volume, sperm motility, concentration, vitality and morphology. Most problems were of volume measurement (weighing method was reported by $17 \%$ of CR and $10 \%$ of NCR) and staining method for sperm morphology (Papanicolau or Diff-Quik were found in $33 \%$ of CR and $23 \%$ of NCR). A three- or four-point grading of sperm motility was used by the majority of respondents; however, $17 \%$ of CR and $37 \%$ of NCR did not use a laboratory counter to tally spermatozoa. Although a haemocytometer method was used by $80 \%$ of laboratories in each group, the improved Neubauer chamber was used only by $42 \%$ of CR and $19 \%$ of NCR. In each group, $24 \%$ of laboratories did not perform a vitality test. Procedural errors and the interchangeable utilization of two or even three methods to analyse a given parameter was observed in both groups. The results indicate a need for standardisation of the methods and continuous, unified training in semen analysis in Polish laboratories.
\end{abstract}

Asian Journal of Andrology (2013) 15, 616-621; doi:10.1038/aja.2013.48; published online 1 July 2013

Keywords: clinical laboratory; compliance; semen analysis; World Health Organization

\section{INTRODUCTION}

On the basis of current world population estimates, one global problem affecting more than 70 million people worldwide is infertility. ${ }^{1}$ According to data provided by four Polish centres for reproduction, the proportion of infertile couples in which the male is responsible may even be as high as $55 \% .^{2}$ Reduced semen quality is commonly claimed to be one of the main signs of male infertility ${ }^{3-5}$ and thus, analysis of semen is recommended as the cornerstone of a male fertility investigation. ${ }^{6,7}$ Semen analysis provides crucial information on the functional status of the seminiferous tubules (sperm production), epididymis, prostate and seminal vesicles (sperm motility and viability) and the patency of the male genital tract, on which clinicians base their initial diagnosis. However, to control possible sources of physiological and methodological variation, and to generate reliable information on semen quality that can be accurately compared across different laboratories, semen analysis should be performed according to generally accepted, international recommendations and standardized procedures. ${ }^{8-11}$

The World Health Organization (WHO) laboratory manual is recognized worldwide as the gold standard for human semen examination. It provides clear guidelines for this analysis and for the reduction of laboratory errors. The manual was first published in 1980. Since then, it has been regularly revised and updated, and the most recent fifth edition appeared in 2010. ${ }^{12}$ Although the WHO manuals have been available for more than 30 years, there are still reports revealing a lack of standardization of the methods used in andrology laboratories and wide variation in the results obtained among laboratory technicians in many countries. ${ }^{8,10,13-16}$

The selection and implementation of methods for semen analysis which are accepted, standardized and validated worldwide should be the basis of good practice in every seminological laboratory. With this aim in mind, the present is a survey of employees of laboratories offering semen analysis in Poland, to ascertain their compliance with WHO recommendations and to determine the variation in the methods used.

\section{MATERIALS AND METHODS}

Design of the study

An anonymous survey was conducted among 55 employees of 55 laboratories in Poland with experience in semen analysis. The respondents were participants of one of 10 editions of a 3-day laboratory workshop on methods in semen analysis according to the latest, fifth

${ }^{1}$ Division of Andrology, Department of Andrology and Reproductive Endocrinology, Medical University of Lodz, 91-425 Lodz, Poland; ${ }^{2}$ Division of Reproductive Endocrinology, Department of Andrology and Reproductive Endocrinology, Medical University of Lodz, 91-425 Lodz, Poland and ${ }^{3}$ Diagnostyka Company Ltd, Medical Laboratories, $31-513$ Cracow, Poland

* These authors contributed equally to this work.

Correspondence: Professor J Slowikowska-Hilczer (jolanta.slowikowska-hilczer@umed.lodz.pl)

Received: 31 January 2013; Revised: 6 March 2013; Accepted: 27 March 2013; Published online: 1 July 2013 
edition of the WHO manual. ${ }^{12}$ All editions of the workshop were held in Polish and were organized in Cracow by the Modern Diagnostic Foundation (Cracow, Poland) in collaboration with the Department of Andrology and Reproductive Endocrinology of Medical University of Lodz (Lodz, Poland), which was the Clinical Training Centre of the European Academy of Andrology, from October 2011 until June 2012.

The questionnaire was designed to obtain information concerning the education level and work experience of respondents, as well as their past participation in workshops on semen analysis and methods and equipment used to analyse the key parameters of the samples in their laboratory. On the basis of the results from a total of 55 respondents, a certified respondents group (CR) was formed from the 17 (31\%) who reported participation in external, certified workshops on semen analysis and a non-certified respondents group (NCR) was formed from the remaining 38 respondents (69\%) who reported having received internal instruction by a senior colleague, or who were self-taught in the methodology of semen analysis.

\section{Compliance with the current WHO recommendations}

The answers for five main parameters, i.e., semen volume measurement and sperm motility, concentration, vitality and morphology, were further analysed as to whether they were compliant with WHO recommendations. The survey was conducted almost 2 years after the online appearance of the fifth edition of the manual in February 2010, (http://whqlibdoc.who.int/publications/2010/9789241547789_eng.pdf). Additionally, on the initiative of the Commission for the MedicalDiagnostic Consensus of the Polish Society of Andrology, and representatives of the Polish Society of Laboratory Diagnostics, the WHO recommendations for semen analysis from this edition were published in Polish in the Journal of Laboratory Diagnostics in the same year. ${ }^{17}$ Every issue of the journal is sent to all diagnostic laboratories throughout Poland. As the last two editions of the WHO manuals were very similar with regard to the basic guidelines for the use of the equipment and the methods in semen analysis that are the focus of this study, it was decided to address the compliance of the laboratories with the recommendations presented in both editions. ${ }^{12,18}$

The volume measurement was considered as compliant when it was estimated by the weighing method. The sperm motility was compliant when respondents reported: (i) counting spermatozoa with the help of laboratory counter or computer-assisted semen analysis (CASA) system and (ii) using a three- or four-point grading scale to distinguish the type of motility. The sperm concentration calculation was considered as compliant when the haemocytometer method was used, with the improved Neubauer or other 100- $\mu \mathrm{m}$-deep chamber (Bürker, Thoma) and the dilution of the semen sample assured spermatozoa immobilisation. When no answer was given on the semen dilution procedure or the type of diluent, the method was recognized as not compliant. The vitality test was compliant when eosin, eosin-nigrosin or hypo-osmotic tests were reported. Sperm morphology was considered compliant when the smears were stained by the Papanicolau, Diff-Quik or Shorr dyes. When sperm vitality or morphology was not analysed at all in any sample, the procedure was rated as not compliant.

\section{RESULTS}

General information about respondents

Within the $\mathrm{CR}, 6 \%$ had a doctorate $(\mathrm{PhD})$ and another 6\% were laboratory technicians with a Bachelor's degree in diagnostics. The remaining respondents (88\%) reported a postgraduate university education (Masters) with diagnostic qualifications. Similarly, within the NCR, the majority of the respondents (87\%) reported having completed a postgraduate degree (Masters) with diagnostic qualifications, while the remainder (13\%) reported having a Bachelor's degree.

Within the CR group, 28\% reported less than 1 year's work experience in semen analysis, $24 \%$ had been working from 1 to 5 years, another $24 \%$ from 5 to 10 years and another $24 \%$ more than 10 years. Within the NCR group, $16 \%$ had been performing semen analysis for less than 1 year, $34 \%$ from 1 to 5 years, $11 \%$ from 5 to 10 years and $39 \%$ more than 10 years.

\section{Semen volume measurements}

The proportion of different methods for semen volume measurements reported by respondents is presented in Table 1 . The most popular method of volume measurement was decanting into a graduated test tube, which was reported by more than $50 \%$ of respondents in both groups. Additionally, one CR and two NCR reported using interchangeably two methods of volume measurement. The CR used weighing and graduated test tube, while NCRs used disposable Pasteur pipette interchangeably with graduated test tube or syringe.

\section{Sperm motility assessment and grading}

The majority of respondents in both groups reported using laboratory counters to assess sperm motility. Nevertheless, in both groups, some respondents performed it without help of any such equipment (Table 2). Again, one member of the CR group and five of the NCR group reported two methods of assessing sperm motility. Of the latter, while two cases reported using a CASA system, three respondents reported performing the test without the help of any equipment. In the remaining cases (one CR and one NCR) motility assessment was performed interchangeably with or without laboratory counters. Surprisingly, $12 \%$ of CR and $13 \%$ of NCR did not specify the method of sperm motility grading. While the majority of both groups graded sperm motility to four grades (rapid progressive, slow progressive, non-progressive, immotile), up to $16 \%$ of NCR reported grading sperm motility into only two grades (progressive and abnormal) (Table 2).

Types of counting chamber and diluents used for estimating sperm concentration

The most popular method used by both groups was a manual, haemocytometric method ( $84 \%$ of CR and $81 \%$ of NCR). The recommended improved Neubauer haemocytometer was more commonly used by the CR group, while the Bürker more commonly by the NCR group (Table 3). Two of the NCR respondents reported using interchangeably two types of haemocytometer: a Bürker or Thoma, and Bürker or improved Neubauer. The remaining respondents reported using a CASA system with no chamber at all ( $4 \%$ of NCR) or with Leya

Table 1 Methods of semen volume measurements reported by the certified and non-certified respondents

\begin{tabular}{lcc}
\hline Volume measurements & $C R$ & $N C R$ \\
& $\mathrm{~N}(\%)$ & $\mathrm{N}(\%)$ \\
\hline Weighing & $3(17)$ & $4(10)$ \\
Decanting into tube & $10(56)$ & $24(60)$ \\
Aspiration into Pasteur pipette & $5(28)$ & $10(25)$ \\
Aspiration into syringe $_{\text {Total }^{\mathrm{a}}}$ & 0 & $2(5)$ \\
\hline
\end{tabular}

Abbreviations: CR, certified respondent; NCR, non-certified respondent.

$N$, number of respondents.

${ }^{a}$ Two methods were reported by one CR and two NCR. 
Table 2 Methods of sperm motility assessment and grading reported by the certified and non-certified respondents

\begin{tabular}{|c|c|c|}
\hline & $\begin{array}{l}C R \\
\mathrm{~N}(\%)\end{array}$ & $\begin{array}{l}N C R \\
N(\%)\end{array}$ \\
\hline \multicolumn{3}{|l|}{ Sperm motility assessment } \\
\hline Laboratory counter & $13(72)$ & $19(44)$ \\
\hline Without the laboratory counter & $3(17)$ & $16(37)$ \\
\hline CASA system & $2(11)$ & $8(19)$ \\
\hline Total $^{\mathrm{a}}$ & 18 & 43 \\
\hline \multicolumn{3}{|l|}{ Sperm motility grading } \\
\hline $\begin{array}{l}4 \text { types } \\
\text { (rapid progressive, slow progressive, non- } \\
\text { progressive, immotile) }\end{array}$ & $11(65)$ & $15(40)$ \\
\hline $\begin{array}{l}3 \text { types } \\
\text { (progressive, non-progressive, immotile) }\end{array}$ & $4(24)$ & $12(32)$ \\
\hline $\begin{array}{l}2 \text { types } \\
\text { (progressive, abnormal) }\end{array}$ & 0 & $6(16)$ \\
\hline not specified & $2(12)$ & $5(13)$ \\
\hline Total & 17 & 38 \\
\hline
\end{tabular}

Abbreviations: CASA, computer-assisted semen analysis; CR, certified respondent; NCR, non-certified respondent;

${ }^{\mathrm{a}}$ Two methods were reported by one CR and five NCR.

or Makler chambers (15\% of CR and 14\% of NCR) (Table 3). Of them, one CR and three NCR also used haemocytometers. The CR used a Thoma, two NCR used an improved Neubauer or a Bürker.

Surprisingly, of those who used $100-\mu \mathrm{m}$-deep haemocytometers, 8 out of 15 CR (53\%) and 22 out of 36 NCR (61\%) reported not making dilutions of the semen sample before loading the chamber, or diluted the semen sample with diluents not assuring spermatozoa immobilization (e.g., $\mathrm{NaCl}$, distilled water, eosin solution, phosphatebuffered saline with fuchsin), or did not answer these questions. One CR, who reported using a Makler chamber without a CASA system used high temperature to immobilize spermatozoa.

\section{Sperm vitality analysis}

The vitality test with eosin alone was the most popular procedure for assessing sperm vitality among respondents from both groups $(76 \%$ of $\mathrm{CR}$ and $68 \%$ of NCR). The use of eosin-nigrosin was reported by only $5 \%$ of NCR. One respondent in this group (3\%) reported performing an analysis of sperm vitality from only a wet preparation, without any staining. None of the respondents in either group reported the use of the hypo-osmotic swelling test. However, almost one fourth of

Table 3 Types of counting chambers used for sperm concentration analysis reported by the certified and non-certified respondents

\begin{tabular}{lcc}
\hline & $C R$ & $N C R$ \\
& $N(\%)$ & $N(\%)$ \\
\hline $\begin{array}{l}\text { Counting chambers without CASA system } \\
\text { Improved Neubauer haematocytometer }\end{array}$ & $8(42)$ & $9(19)$ \\
Bürker haematocytometer & $4(21)$ & $21(48)$ \\
Thoma haematocytometer & $3(16)$ & $6(14)$ \\
$\quad$ Makler & $1(5)$ & 0 \\
Counting chambers with CASA system & & \\
Leya & $2(10)$ & $3(7)$ \\
Makler & $1(5)$ & $3(7)$ \\
none & 0 & $2(4)$ \\
Total & & \\
\hline
\end{tabular}

Abbreviations: CASA, computer-assisted semen analysis; CR, certified respondent;

${ }^{a}$ Two types of counting chamber were reported by two CR and six NCR. respondents in each group did not analyse this parameter at all ( $24 \%$ of CR and $24 \%$ of NCR).

\section{Staining methods for sperm morphology evaluation}

Only $33 \%$ of CR and $23 \%$ of NCR reported using methods recommended by the two most recent editions of the WHO manual (Table 4). ${ }^{12,18}$ Surprisingly, $11 \%$ of CR and 7\% of NCR reported using only the wet preparation, without any staining, for sperm morphology evaluation, $4 \%$ of NCR reported using eosin staining and $7 \%$ of the NCR members did not analyse this parameter at all. The remaining respondents used primarily the Giemsa-Wright method (Table 4). One CR respondent and three of the NCR group reported using two types of staining, and one NCR group member even noted using three types. The CR reported using Giemsa-Wright or Diff-Quik staining. Two of the three NCR reported using either the Papanicolau or Giemsa-Wright methods, while the third one used Diff-Quik and SpermacStain. The last respondent, who reported three methods, used Giemsa-Wright or eosin staining under $\times 1000$ magnification in the light microscope or no staining at all.

\section{Compliance with WHO manual recommendations}

Only one of the CR group performed all five parameters in compliance with the WHO recommendations. For the NCR, at least one parameter was performed with methods incompatible with the WHO recommendations and, in $8 \%$ of them, the lack of compliance extended to all five parameters (Table 5). In both groups, the most prevalent inconsistency was in volume measurement and the staining for sperm morphology. The inconsistency in sperm motility was much higher within the NCR group (50\% vs. 6\% of CR) (Table 5).

\section{DISCUSSION}

The principle of good practice in laboratories is the implementation of standardized, validated methods and equipment for the evaluation of the defined parameters. In consequence, it assures a proper basis for achieving an accurate, reliable and controlled service in the laboratory and its accreditation. ${ }^{10,13,19}$ For more than 30 years, the WHO has attempted to improve the quality of semen analysis by publishing manuals providing a detailed description of standardized methods and procedures for the evaluation of semen analysis. ${ }^{12,18,20}$ Unfortunately, adherence to these guidelines is sometimes selective

Table 4 Staining methods for sperm morphology assessment reported by the certified and non-certified respondents

\begin{tabular}{lcc}
\hline Staining methods & $C R$ & $N C R$ \\
& $N(\%)$ & $N(\%)$ \\
\hline Papanicolau & $2(11)$ & $4(9)$ \\
Diff-Quik & $4(22)$ & $6(14)$ \\
Other & $10(56)$ & $27(63)$ \\
No staining method & $2(11)$ & $3(7)$ \\
Parameter not analysed & 0 & $3(7)$ \\
Total & 18 & 43 \\
Other staining methods used & & \\
$\quad$ Giemsa-Wright & $8(80)$ & $19(70)$ \\
Spermac Stain & $0)$ & $2(7)$ \\
Test Simplets & $2(20)$ & $2(7)$ \\
Eosin & 0 & $1(4)$ \\
Not specified & 0 & $3(11)$ \\
\hline
\end{tabular}

Abbreviations: CR, certified respondent; NCR, non-certified respondent;

${ }^{a}$ Two types of staining method were reported by one CR and three NCR and three types by one NCR. 
Table 5 Lack of compliance with WHO recommendations in the parameters reported by the certified and non-certified respondents

\begin{tabular}{lcc}
\hline & $C R$ & $N C R$ \\
& $N(\%)$ & $N(\%)$ \\
\hline $\begin{array}{l}\text { Number of non-compliant parameters } \\
\text { O parameter }\end{array}$ & $1(6)$ & 0 \\
1 parameter & $4(24)$ & $4(10)$ \\
2 parameters & $5(29)$ & $14(37)$ \\
3 parameters & $4(24)$ & $12(32)$ \\
4 parameters & $3(18)$ & $5(13)$ \\
all parameters & 0 & $3(8)$ \\
Total & 17 & 38 \\
Type of non-compliant parameter & & \\
Volume measurement & $14(82)$ & $34(89)$ \\
Sperm motility & $1(6)$ & $19(50)$ \\
Sperm concentration & $8(41)$ & $22(58)$ \\
Sperm vitality & $4(24)$ & $10(26)$ \\
Sperm morphology & $12(71)$ & $29(76)$ \\
\hline
\end{tabular}

Abbreviations: CR, certified respondent; NCR, non-certified respondent. $N$, number of respondents.

and laboratories adopt not all the recommended methods for their seminological service, which may result in difficulties in interpreting and comparing the results of semen analysis. ${ }^{10,21-23}$

To our knowledge, our study is the first to analyse the existing variations in the methods used in seminological laboratories in Poland and Central Europe. It must be noted that all the respondents participating in our survey demonstrated an intention to improve their laboratory skills if they attended the workshop the theme of which concerned the implementation of the new recommendations for semen analysis published in the fifth edition of the WHO manual. ${ }^{12}$ It may only be speculated that many other laboratories operate but do not show such a desire. As such, the analysis of the methods used in the laboratories evaluated in the present study may be regarded as an overly optimistic picture of the seminological service in Poland.

Although a better selection of methods compliant with the WHO recommendations ${ }^{12,18}$ was expected in laboratories from which technicians reported participation in external, certified workshops for procedures of semen analysis, surprisingly little difference in the selection of the methods used for semen analysis was observed between the CR and NCR groups. In both groups, some examples of gross methodological inadequacy were observed in the analysis of a given parameter, suggesting that a poor service is offered in some laboratories, which must result in unreliable data. Procedural errors, such as the analysis of sperm morphology from a wet preparation or eosin staining, the estimation of sperm concentration in a haematocytometer but without making semen dilutions or without immobilization of spermatozoa, not using laboratory counters for sperm motility assessment or distinguishing only two types of sperm movement were reported. It is also difficult to explain the phenomenon of interchangeably using two, or even three methods that might not be compatible with each other, for the evaluation of a given parameter. A possible explanation is the personal preference of a laboratory technician, which should not be acceptable in any diagnostic centre. ${ }^{13}$ This methodological inadequacy, as well as the use of multiple methods for the evaluation of a single parameter, may result from the lack of top-down directives from the supervisory authorities or a lack of internal and external quality control in laboratories. ${ }^{14,19,24}$

It is alarming that of the 55 respondents representing different laboratories in Poland, only in one case were the methods used to evaluate the principal semen parameters compliant with the guidelines presented in both of the last editions of the WHO manual.

It is surprising that semen volume measurement and the selection of staining methods for evaluation of sperm morphology seem to be the most problematic procedures among the respondents, regardless of whether or not they were certified in semen analysis techniques. The lack of compliance with the recommended WHO methods in both groups reached more than $80 \%$ for volume measurement and more than $70 \%$ for the staining methods for sperm morphology evaluation. There are many reports clearly indicating that methods of volume measurement utilizing aspiration or decanting the semen sample into scaled pipettes, syringes, tubes or cylinders result in the underestimation of results to an extent of between 0.3 and $0.9 \mathrm{ml}^{25-28}$ However, both methods, decanting into a graduated cylinder, and aspiration into a wide-mouthed pipette, were only described in an outdated WHO manual from $1992 .{ }^{20}$ Hence, the use of those methods by such a large group of our respondents suggests that in many laboratories the semen analysis procedure has been passed on through the years without any effort to modernize it, or with only some modernization of selected parts. Precise volume measurement is essential because it allows the calculation of the total number of spermatozoa and nonsperm cells. The total number of living spermatozoa with progressive motility or normal morphology in the whole ejaculate is of biological significance and may have clinical implications. The choice of the 'decanting' or 'aspirating' method cannot be explained on the basis of simplicity or increased safety when compared with the use of weighing, or by the cost of additional equipment. A simple laboratory balance is unlikely to outstrip the financial means of the laboratory. One explanation could be that there are still laboratories where the sample is collected by patient into a container not provided by the laboratory, and thus not pre-weighed. This situation may be connected with poor quality assurance in the laboratory. ${ }^{24}$

Sperm morphology assessment, when performed according to welldefined procedures, gives data of high predictive value for male fertility potential. ${ }^{29}$ However, in our study, $7 \%$ of the NCR respondents did not analyse this parameter at all. For years, sperm morphology has been recognized as probably the most confusing and subjective area of semen analysis. ${ }^{10,30-32}$ It is important to implement the recommended, generally-accepted staining methods for semen smear evaluation to minimize the possible analytical errors at this level. Although there are many staining methods for the evaluation of cell morphology, not all are suited to spermatozoa. The morphological characteristics of the spermatozoa stained by them have not been appropriately described and validated, and the obtained results may give statistically significant deviations from recommended methods. ${ }^{33}$ Although for years, the WHO has recommended the Papanicolau staining as the one that best provides good clarity for smear viewing and colour differentiation between the regions of spermatozoa, ${ }^{12,18,34}$ it was used only by about $10 \%$ of respondents in each group, probably because the method is time consuming. In the last two editions of the WHO manual, ${ }^{12,18}$ the Diff-Quik, simpler staining method, ${ }^{35}$ is presented as the alternative to Papanicolau staining; however, this method again did not predominate in either group of respondents. The most prevalent staining method turned out to be the Giemsa-Wright method, a staining method dedicated to the differentiation of blood cells. However, with this approach, seminal plasma stains vivid purple, giving a background that obscures much of the fine detail of sperm morphology. ${ }^{34,36}$ The popularity of this staining procedure might result from its availability in each diagnostic laboratory performing blood tests, leading to its consequent adoption for sperm staining, 
especially since it was recommended in the outdated WHO manual from $1992 .{ }^{20}$ Unfortunately, this observation confirms that for years most of the laboratories surveyed have not updated their seminological service. Probably also for this reason, some respondents reported using no staining method for sperm morphology evaluation, utilizing only a wet preparation for this purpose, a method regarded as acceptable in the above-mentioned outdated manual, but not in the most recent editions. ${ }^{37,38}$ It is also difficult to explain why, in the same laboratory, recommended methods (Papanicolau or Diff-Quik) were used interchangeably with ones which were not recommended (e.g. Giemsa-Wright staining). Could this choice be connected with the personal preference of the laboratory technician, the characteristics of the semen sample, or with the number of samples to be analysed by a single technician during 1 day? Whatever the cause, such practices should be avoided.

For sperm vitality evaluation, it is alarming that in each group almost one fourth of the respondents reported not analysing this parameter in any sample. The determination of the percentage of viable spermatozoa, especially when their motility is strongly affected, may be helpful in indicating whether the spermatozoa could be used for in vitro fertilisation or intracytoplasmic sperm injection. Although the last two editions of the WHO manual present three methods of evaluating sperm vitality, publications have demonstrated that the use of the eosin-nigrosin test is preferable to that of the eosin test. ${ }^{39}$ However, the latter would seem to be the most popular in laboratories in Poland, perhaps because of its simplicity.

The haemocytometer method for the evaluation of sperm concentration, utilizing $100-\mu \mathrm{m}$-deep chambers with a diluted semen sample assuring sperm immobilization, is recommended by the WHO. In our study, about $50 \%$ of respondents in each group did not meet those recommendations. It is also surprising that the improved Neubauer chamber was used by about only $40 \%$ of CR respondents and only $19 \%$ of NCR, especially when the detailed description of the counting procedure with this chamber has been published in subsequent editions of the manual. ${ }^{12,18,20}$ Thus, the implementation of these procedures would not have presented any difficulties. Moreover, it has been emphasized that any variation in the standardized procedure (e.g., by the use of any other chamber) requires its validation, incorporating appropriate statistical analysis and comparison of obtained results with the standardized method. ${ }^{12,18,19}$ The popularity of Thoma or Bürker chambers, especially among the NCR group, might again be connected with the 'tradition' of using these types of haemocytometer for the blood tests. This observation confirms the lack of modernization within the seminological service. The use of any other counting chamber (e.g., Makler) for manual sperm concentration analysis has been shown to give discrepancies with the results obtained from the improved Neubauer chamber, and these may have consequences in clinical decisions. ${ }^{40,41}$ Other studies clearly demonstrate the differences in results of sperm concentration when using the recommended haemocytometer method, usually with an improved Neubauer haemocytometer, and a CASA system, utilizing usually $20-\mu \mathrm{m}$-deep chambers. ${ }^{42-45}$

The methods used for evaluation of sperm motility by members of the NCR group were of poor quality. When the compliance with the WHO recommendations is considered $50 \%$ of NCR did not meet the recommended standards, in comparison with only $6 \%$ of CR. Sperm motility is recognized as one of the most difficult parameters to standardize and to learn by the technician, and consequently large inter- and intra-laboratory differences can be seen in the results. ${ }^{30,46}$ Performing sperm counts and categorizing the type of motility without help of any device is a challenge. The CASA system is recognized as a more objective method for sperm motility evaluation; however, its availability is limited because of its cost.

In conclusion, our results present the existence of (i) differences in seminological services in the methods and equipment used; (ii) gross methodological inadequacies; and (iii) inconsistency with the WHO recommendations in Polish laboratories. These may reflect: (i) the poor quality of training or workshops dedicated to this specific diagnostic area; (ii) the poor implementation of the standards into the laboratory service; (iii) the poor quality control of seminological laboratories; ${ }^{24}$ and (iv) the lack of obligatory directives for semen analysis. We have revealed that the seminological service is neglected in Poland and needs serious improvement. These results indicate a need for standardisation of the methods and continuous, unified training on semen analysis in Polish laboratories.

\section{AUTHOR CONTRIBUTIONS}

RWJ, KM and JSH participated in the conception of the study and the questionnaire design. KM, RWJ, EF, LB and EO distributed and collected the questionnaire. RWJ and KM analysed the questionnaires, interpreted the data and drafted the manuscript. JSH, KM, EO, EF and LB revised critically the manuscript. All authors read and approved the final manuscript.

\section{COMPETING FINANCIAL INTERESTS}

The authors declare that there are no competing financial interests.

\section{ACKNOWLEDGMENTS}

This work was supported by Medical University of Lodz (grant nos. 503/11089-02/503-1 and 503/1-1089-03/503-1). The authors thank Mr Edward Lowczowski (MA), a native speaker, for manuscript language correction.

Boivin J, Bunting L, Collins JA, Nygren KG. International estimates of infertility prevalence and treatment-seeking: potential need and demand for infertility medical care. Hum Reprod 2007; 22: 1506-12.

2 Bablok L, Dziadecki W, Szymusik I, Wolczynski S, Kurzawa R et al. Patterns of infertility in Poland-multicenter study. Neuro Endocrinol Lett2011; 32: 799-804.

3 de Kretser DM. Male infertility. Lancet 1997; 349: 787-90.

4 Franken DR, Oehninger S. Semen analysis and sperm function testing. Asian J Androl 2012; 14: 6-13.

5 Bungum M, Bungum L, Giwercman A. Sperm chromatin structure assay (SCSA): a tool in diagnosis and treatment of infertility. Asian J Androl 2011; 13: 69-75.

6 Nallella KP, Sharma RK, Aziz N, Agarwal A. Significance of sperm characteristics in the evaluation of male infertility. Fertil Steril 2006; 85: 629-34.

7 Barratt CL. Semen analysis is the cornerstone of investigation for male infertility. Practitioner 2007; 251: 8-10, 12, 15-7.

8 Keel BA. Quality control, quality assurance, and proficiency testing in the andrology laboratory. Arch Androl 2002; 48: 417-31.

9 Alvarez C, Castilla JA, Martinez L, Ramirez JP, Vergara F et al. Biological variation of seminal parameters in healthy subjects. Hum Reprod 2003; 18: 2082-8.

10 Riddell D, Pacey A, Whittington K. Lack of compliance by UK andrology laboratories with World Health Organization recommendations for sperm morphology assessment. Hum Reprod 2005; 20: 3441-5.

11 Castilla JA, Alvarez C, Aguilar J, Gonzalez-Varea C, Gonzalvo MC et al. Influence of analytical and biological variation on the clinical interpretation of seminal parameters. Hum Reprod 2006; 21: 847-51.

12 WHO. WHO Laboratory Manual for the Examination and Processing of Human Semen. Cambridge: Cambridge University Press; 2010.

13 Keel BA. How reliable are results from the semen analysis? Fertil Steril 2004; 82: 41-4.

14 Pacey AA. Is quality assurance in semen analysis still really necessary? A view from the andrology laboratory. Hum Reprod 2006; 21: 1105-9.

15 Pacey AA. Are sperm counts declining? Or did we just change our spectacles? Asian J Androl 2013; 15: 187-90.

16 Mallidis C, Cooper TG, Hellenkemper B, Lablans M, Uckert F et al. Ten years' experience with an external quality control program for semen analysis. Fertil Steril 2012; 98: 611-6.e4.

17 Bergier L, Bakalczuk S, Fracki S, Marchlewska K, Piasecka M et al. Semen analysis the manual method. Standards according to WHO guidelines from 2010, prepared by the Commission for the Medical-Diagnostic Consensus of the Polish Society of 
Andrology (PTA) with representatives of the Polish Society of Laboratory Diagnostics (PTDL). J Lab Diagn 2010; 46: 161-70. Polish.

18 WHO. WHO Laboratory Manual for the Examination of Human Semen and SpermCervical Mucus Interaction. Cambridge: Cambridge University Press; 1999.

19 Pike IL. Accreditation and the use of validated/recognised methods to analyse human semen. Reprod Biol 2011; 11(Suppl 3): 5-15.

20 WHO. WHO Laboratory Manual for the Examination of the Human Semen and SpermCervical Mucus Interaction. Cambridge: Cambridge University Press; 1992.

21 Badenoch DF, Evans SJ, McCloskey DJ. Sperm density measurement: should this be abandoned? Br J Urol 1989; 64: 521-3.

22 Neuwinger J, Behre HM, Nieschlag E. External quality control in the andrology laboratory: an experimental multicenter trial. Fertil Steril 1990; 54: 308-14.

23 Keel BA, Stembridge TW, Pineda G, Serafy NT Sr. Lack of standardization in performance of the semen analysis among laboratories in the United States. Fertil Steril 2002; 78: 603-8.

24 Pacey AA. Quality assurance and quality control in the laboratory andrology. Asian J Androl 2010; 12: 21-5.

25 Brazil C, Swan SH, Drobnis EZ, Liu F, Wang C et al. Standardized methods for semen evaluation in a multicenter research study. J Androl 2004; 25: 635-44.

26 Iwamoto T, Nozawa S, Yoshiike M, Hoshino T, Baba K et al. Semen quality of 324 fertile Japanese men. Hum Reprod 2006; 21: 760-5.

27 Cooper TG, Brazil C, Swan SH, Overstreet JW. Ejaculate volume is seriously underestimated when semen is pipetted or decanted into cylinders from the collection vessel. J Androl 2007; 28: 1-4.

28 Matson PL, Myssonski K, Yovich S, Morrison L, Irving J et al. The density of human semen and the validation of weight as an indicator of volume: a multicentre study. Reprod Biol 2010; 10: 141-53.

29 Menkveld R, Holleboom CA, Rhemrev JP. Measurement and significance of sperm morphology. Asian J Androl 2011; 13: 59-68.

30 Franken DR, Aneck-Hahn N, Lombaard C, Kruger TF. Semenology training programs: 8 years' experience. Fertil Steril 2010; 94: 2615-9.

31 Yao KS, Zhang XZ, Wu Y. Assessment of sperm morphology without quality control may be meaningless for clinicians. Asian J Androl 2010; 12: 607-8.

32 Ariagno JI, Curi SM, Chenlo P, Repetto HE, Pugliese MN et al. Our experience in sperm morphology assessment. Asian J Androl 2011; 13: 201-2.

33 Henkel R, Schreiber G, Sturmhoefel A, Hipler UC, Zermann DH et al. Comparison of three staining methods for the morphological evaluation of human spermatozoa. Fertil Steril 2008; 89: 449-55.
34 Rothmann SA, Reese AA. Semen analysis: the test techs love to hate. MLO Med Lab Obs 2007; 39: 18-27.

35 Kruger TF, Ackerman SB, Simmons KF, Swanson RJ, Brugo SS et al. A quick, reliable staining technique for human sperm morphology. Arch Androl 1987; 18: 275-7.

36 Cipak A, Stanic P, Duric K, Serdar TE. Sperm morphology assessment according to WHO and strict criteria: method comparison and intra-laboratory variability. Biochem Med 2009; 19: 97-4.

37 Meschede D, Keck C, Zander M, Cooper TG, Yeung CH et al. Influence of three different preparation techniques on the results of human sperm morphology analysis. Int J Androl 1993; 16: 362-9.

38 Oral E, Yetis O, Elibol F, Senol H, Irez T et al. Assessment of human sperm morphology by strict criteria: comparison of wet preparation versus stained with the modified DiffQuik method. Arch Androl 2002; 48: 307-14.

39 Cooper TG, Hellenkemper B. Method-related estimates of sperm vitality. J Androl 2009; 30: 214-8

40 Tomlinson M, Turner J, Powell G, Sakkas D. One-step disposable chambers for sperm concentration and motility assessment: how do they compare with the World Health Organization's recommended methods? Hum Reprod 2001; 16: 121-4.

41 Marchlewska K, Filipiak E, Oszukowska E, Walczak-Jedrzejowska R, Kula K et al. Comparison of the precision in determination of human sperm concentration between an improved Neubauer haematocytometer and Makler counting chamber. Clin Exp Med Lett 2010; 51: 131-4. Polish.

42 Spiropoulos J. Computerized semen analysis (CASA): effect of semen concentration and chamber depth on measurements. Arch Androl 2001; 46: 37-42.

43 Douglas-Hamilton DH, Smith NG, Kuster CE, Vermeiden JP, Althouse GC. Capillaryloaded particle fluid dynamics: effect on estimation of sperm concentration. J Androl 2005; 26: 115-22.

44 Douglas-Hamilton DH, Smith NG, Kuster CE, Vermeiden JP, Althouse GC Particle distribution in low-volume capillary-loaded chambers. J Androl 2005; 26: 107-14.

45 Vested A, Ramlau-Hansen CH, Bonde JP, Thulstrup AM, Kristensen SL et al. A comparison of conventional and computer-assisted semen analysis (CRISMAS software) using samples from 166 young Danish men. Asian J Androl 2011; 13 453-8.

46 Auger J, Eustache F, Ducot B, Blandin T, Daudin M et al. Intra- and inter-individual variability in human sperm concentration, motility and vitality assessment during a workshop involving ten laboratories. Hum Reprod 2000; 15: 2360-8. 